\title{
Carriers of continuous measures in a Hilbertian norm
}

\author{
By \\ Yasuo UMEMURA
}

Summary. From the standpoint of the theory of measures on the dual space of a nuclear space, we discuss the carrier of Wiener measure, regarding it as a measure on $\left(D^{\prime}\right)$ (=Schwartz's space of distributions). This may be contrasted with the usual treatment which regards it as a measure on the space of paths.

It is shown that for $\alpha>\frac{1}{2}$, integral operator $I_{a}$ is nuclear on $L^{2}((0,1))$ ( $\left.\equiv H_{0}\right)$. Using this fact, we see that Wiener measure lies on the space $I_{B}\left(H_{0}\right) \quad\left(\beta<\frac{1}{2}\right)$ which consists of Hölder continuous functions of the $\beta$-th order in the sense of $L^{2}$. This result is true for any measure whose characteristic functional is continuous on $L^{2}((0,1))$.

\section{$\S 1$. Nuclear operators and carriers of measures}

Let $H_{0}$ be a real Hilbert space with the scalar product $\langle\xi, \eta\rangle_{0}$, and $L$ be its subspace which is dense and nuclear in $H_{0}$. It means that there exists a complete orthonormal system $\left\{\xi_{k}\right\}$ in $H_{0}$ and a sequence of positive numbers $\left\{a_{k}\right\}$ such that $\sum_{k=1}^{\infty} a_{k}^{2}<\infty$ and the norm $\|\xi\|_{1}^{2}=\sum_{k=1}^{\infty} \frac{\left\langle\xi, \xi_{k}\right\rangle_{0}^{2}}{a_{k}^{2}}$ is continuous in the proper topology of $L$. R. A. Minlos proved that for any positive definite and continuous functional $\chi(\xi)$ on $H_{0}$, there exists a measure $\mu$ on $L^{*}$ such that for any $\xi \in L$,

$$
\chi(\xi)=\int \exp [i \xi(x)] d \mu(x),
$$

$\chi(\xi)$ is called the characteristic functional of $\mu$. 
Suppose that both $L_{1}$ and $L_{2}$ are dense and nuclear in $H_{0}$, and that $L_{1} \subset L_{2}$. Then, for given $\chi(\xi)$, we can construct measures $\mu_{1}$ on $L_{1}^{*}$ and $\mu_{2}$ on $L_{2}^{*}$. Identifying them, we can say that the measure $\mu_{1}$ on $L_{1}^{*}$ has the carrier in $L_{2}^{*}\left(\subset L_{1}^{*}\right)$.

Now, let $L$ be a fixed dense and nuclear subspace of $H_{0}$, and $\chi(\xi)$ be a fixed positive definite and continuous functional on $H_{0}$. We shall discuss the carrier of the measure $\mu$ which is defined on $L^{*}$, corresponding to $\chi(\xi)$.

Consider an operator $T$ which satisfies the following conditions;

(1) $T$ is defined and nuclear on $H_{0}$.

(Nuclearity of $T$ is defined by $\sum_{k=1}^{\infty}\left\|T \xi_{k}\right\|_{0}^{2}<\infty$, where $\left\{\xi_{k}\right\}$ is a complete orthonormal system of $H_{0}$ )。

(2) $T$ is one-to-one.

(3) The image $T\left(H_{0}\right)$ includes $L$, and $T^{-1}(L)$ is dense in $H_{0}$.

(4) $T \xi_{j} \rightarrow 0$ in the topology of $L$ implies that $\left\|\xi_{j}\right\|_{0} \rightarrow 0$. In other words, the inverse operator $T^{-1}$ maps $L$ into $H_{0}$ continuously。

Proposition 1 . For any operator $T$ which satisfies (1) (4), the measure $\mu$ (which corresponds to the given $\chi(\xi)$ ) has the carrier in $T^{-1 *}\left(H_{0}\right)$, where $T^{-1 *}$ means the adjoint operator of $T^{-1} \cdot\left(T^{-1 *}\right.$ maps $H_{0}^{*} \simeq H_{0}$ into $L^{*}$ continuously).

Proøf. For $\xi=T \eta \in T\left(H_{0}\right)$, define the norm $\|\xi\|_{1}$ by $\|\xi\|_{1}=\left\|T^{-1} \xi\right\|_{0}$ 。 By this norm, $T\left(H_{0}\right)$ becomes a Hilbert space which we denote by $H_{1}$. From the conditions (4) and (1), the topology of $H_{1}$ is weaker than that of $L$, but stronger than that of $H_{0}$. Hence, $H_{0} \simeq H_{0}^{*} \subset$ $H_{1}^{*} \subset L^{*}$.

From the condition (1), $H_{1}$ is nuclear in $H_{0}$, so that the measure $\mu$ has the carrier in $H_{1}^{*}$. Thus, only remained to prove is that $H_{1}^{*}=T^{-1 *}\left(H_{0}\right)$.

If $x=T^{-1 *} y \in T^{-1 *}\left(H_{0}\right)$. then $(\xi, x)=\left(\xi, T^{-1 *} y\right)=\left(T^{-1} \xi, y\right)$ is continuous on $H_{1}$ so that $x \in H_{1}^{*}$. Conversely, if $x \in H_{1}^{*}$, then the relation $(\xi, x)=\left(T^{-1} \xi, y\right)$ determines uniquely $y \in H_{0}$ and $x=T^{-1 *} y \in T^{-1 *}\left(H_{0}\right)$. (q.e.d.)

Since $\mu$ is completely additive, we get the following corollary.

Corollary. If each of operators $T_{n}(n=1,2, \cdots)$ satisfies the 
conditions $(1) \sim(4)$, then the measure $\mu$ has the carrier in $\bigcap_{n=1}^{\infty} T_{n}^{-1 *}\left(H_{0}\right)$.

\section{§ 2. Integral operators}

We shall apply the result of $\S 1$ to the case of $H_{0}=L^{2}((0,1))$ and $L=\mathscr{D}(0,1))$. Namely, we shall discuss the carrier of a measure $\mu$ on $\left(D^{\prime}\right)$ whose characteristic functional is continuous in $L^{2}((0,1))$.

At first, define the integral operator $I_{\alpha}$ as follows;

$$
I_{\alpha} f(t)= \begin{cases}\int_{0}^{t} \frac{(t-s)^{\alpha-1}}{\Gamma(\alpha)} f(s) d s . & (\alpha<0) \\ \frac{d^{m}}{d t^{m}} I_{\alpha^{\circ} \vdash m} f(t) . & (\alpha \leq 0)\end{cases}
$$

The main properties of this operator are;

a) For any real $\alpha$, it is defined as a operator on $\mathscr{D}((0,1))$, and maps $\mathcal{D}$ into $L^{2}((0,1))$ continuously.

b) For any real $\alpha$, it is defined as a operator on $L^{2}$ which maps $L^{2}$ into $\left(\mathscr{D}^{\prime}\right)$ continuously.

c) For $\alpha>\frac{1}{2}$, it maps $L^{2}$ into $L^{2}$ continuously.

d) For any $\alpha$ and $\beta$, the relation: $I_{\beta} I_{\alpha}=I_{\alpha \mid \beta}$ holds on $\mathcal{D}$. If $\alpha>\frac{1}{2}$, it holds on $L^{2}$ also.

Proposition 2. For $\alpha>\frac{1}{2}$, the operator $I_{a}$ satisfies the conditions $(1) \sim(4)$ of $\S 1$.

Proof. It is easily seen that $I_{\infty}$ satisfies (2) (4). (Especially, $\left.I_{\infty}^{-1}=I_{-\infty}\right)$. So, we shall only prove the nuclearity of $I_{\alpha}$.

Let $\left\{f_{n}(t)\right\}$ be a complete orthonormal system of $L^{2}((0,1))$. It is sufficient to show that $\sum_{n} \int_{0}^{1}\left|I_{\alpha} f_{n}(t)\right|^{2} d t<\infty$, namely,

$$
\sum_{n} \int_{0}^{1}\left[\int_{0}^{t} \frac{(t-s)^{\alpha-1}}{\Gamma(\alpha)} f_{n}(s) d s \int_{0}^{t} \frac{(t-r)^{\alpha-1}}{\Gamma(\alpha)} \overline{f_{n}(r)} d r\right] d t<\infty
$$

By Fubini's theorem, we can rewrite the left hand side of (3) into the form;

$$
\sum_{n} \int_{s=0}^{1} \int_{r=0}^{1} F(s, r) f_{n}(s) \overline{f_{n}(r)} d s d r
$$


where

$$
F(s, r)=\frac{1}{\Gamma(\alpha)^{2}} \int_{\operatorname{Max}(s, r)}^{1}(t-s)^{\alpha-1}(t-r)^{\alpha-1} d t
$$

We see that $F(s, r)$ is a continuous function of $(s, r)$ for $\alpha>\frac{1}{2}$. On the other hand, for instance, putting $f_{n}(s)=\exp (2 \pi i n s)$, we have

$$
\sum_{n} f_{n}(s) \overline{f_{n}(r)}=\sum_{n} \exp (2 \pi i n(s-r)) \overrightarrow{(n \rightarrow \infty)} \delta(s-r)
$$

in $C^{\prime}$ (= the dual of the space of continuous functions).

Hence, the left hand side of (3) is equal with

$$
\begin{aligned}
\int_{0}^{1} F(s, s) d s & =\frac{1}{\Gamma(\alpha)^{2}} \int_{0}^{1}\left[\int_{s}^{1}(t-s)^{2 \alpha-2} d t\right] d s \\
& =\frac{1}{2 \alpha(2 \alpha-1)} \frac{1}{\Gamma(\alpha)^{2}}<\infty
\end{aligned}
$$

From Prop. 1 and Prop. 2, we see that the carrier of the measure $\mu$ lies in $I_{-\infty}^{*}\left(H_{0}\right)$. It is easy to see $I_{-\infty}^{*}=P I_{-\alpha} P$, where $P f(t)=f(1-t)$. However, if at the first step we change the variable $t$ into $1-t$, we need not consider the effect of $P$. Thus, we can say that the carrier of $\mu$ lies in $I_{-a}\left(H_{0}\right)$.

Remark that $I_{-\alpha}=\frac{d}{d t} I_{1-\alpha}$, Therefore, putting $\beta=1-\alpha$, we get the following result.

Proposition 3. Suppose that the characteristic functional of a measure $\mu$ is continuous in $L^{2}((0,1))$, then the carrier of $\mu$ lies in the whole of derivatives of $I_{\beta}\left(H_{0}\right)$ for any $\beta<\frac{1}{2}$, hence in the whole of derivatives of $\bigcap_{n} I_{\beta_{n}}\left(H_{0}\right)$ where $\beta_{n} \uparrow \frac{1}{2}$.

Here, we consider derivatives in the sense of distributions.

\section{§3. Hölder continuity}

Proposition 4. Even for $0<\beta \leqq \frac{1}{2}, I_{\beta}$ is a continuous operator from $L^{2}=H_{0}$ into itself, though $I_{\beta} f(t)$ can be defined only for almost all $t$.

Proof. If $0<\beta \leqq \frac{1}{2}$ and $f(t) \in H_{0}=L^{2}, I_{\beta} f(t)$ can not be defined in the pointwise way, but it can be defined for almost all $t$, because 
the function $\frac{(t-s)^{\beta-1}}{\Gamma(\beta)} f(s)(t \geqq s)$ is integrable with respect to two variables $(s, t)$. Moreover for any $g(t) \in H_{0}$, we have

$$
\begin{aligned}
\int_{0}^{1}|g(t)| \int_{0}^{t} \frac{(t-s)^{\beta-1}}{\Gamma(\beta)}|f(s)| d s d t & \leq \frac{1}{\Gamma(\beta)} \iint_{s+t \leqq 1}|g(t+s)||f(s)| t^{\beta-1} d t d s \\
& \leqq \frac{1}{\Gamma(\beta)}\|g\|_{0}\|f\|_{0} \frac{1}{\beta}
\end{aligned}
$$

Hence, $\left\|I_{\beta} f(t)\right\|_{0} \leqq \frac{1}{\beta \Gamma(\beta)}\|f\|_{0}$.

In general, for $g(t) \in L^{2}((0,1))$, the shift operator $\tau_{h} g(t)=g(t+h)$ has no meaning, since $g(t+h)$ belongs to $L^{2}((-h, 1-h))$, but not to $L^{2}((0,1))$. However, if $g(t) \in I_{\beta}\left(H_{0}\right)$, we can define the shift operator, because the definition (2) of $I_{a}$ can be applied for $t>1$. (For $t<0$, we put $\left.I_{\alpha} f(t)=0\right)$.

Proposition 5. If $g(t) \in \bigcap_{n} I_{\beta_{n}}\left(H_{0}\right)$ where $\beta_{n} \uparrow \frac{1}{2}$, then for any $\beta<\frac{1}{2}, g(t)$ is Hölder continuous in the sense of $L^{2}$. Namely;

$$
\frac{\tau_{h} g(t)-g(t)}{h^{\beta}} \underset{(h \rightarrow 0)}{\longrightarrow} 0 \text { in } L^{2}((0,1)) .
$$

Proof. Since $g(t)=I_{B_{n}} f_{n}(t)$ where $f_{n}(t) \in L^{2}=H_{0}$, in a similar way with (4) we have for any $\varphi(t) \in L^{2}$,

$$
\begin{aligned}
& \int_{0}^{1}|\varphi(t)||g(t+h)-g(t)| d t \\
\leqq & \frac{1}{\Gamma\left(\beta_{n}\right)}\left\|f_{n}\right\|\left\|_{0}\right\| \varphi\left\|_{0} \int_{-|h|}^{1}\left|t_{+}^{\beta_{n}-1}-(t+|h|)^{\beta_{n}-1}\right| d t \leqq \frac{2|h|^{\beta_{n}}}{\beta_{n} \Gamma\left(\beta_{n}\right)}\right\| f_{n}\|\|_{0}\|\varphi\|_{0} .{ }^{1)}
\end{aligned}
$$

Thus, for given $\beta<\frac{1}{2}$, choose $\beta_{n}>\beta$, then

$$
\left\|\frac{\tau_{h} g(t)-g(t)}{h^{\beta}}\right\|_{0} \leqq \text { const. } \times|h|^{\beta_{n}-\beta} \underset{(h \rightarrow 0)}{\longrightarrow} 0 .
$$

Since $g(t)$ is defined only for almost all $t$, the concept of pointwise Hölder continuity loses its meaning. This fault can not be removed as long as we regard $g(t)$ as a distribution. Along this line, we get only the following proposition.

1) $t_{+}^{\beta}=t^{\beta}$ for $t>0,=0$ for $t<0$. 
Proposition 6. If a sequence $\left\{h_{k}\right\}$ satisfies 1) $h_{k} \rightarrow 0$, and 2) $\varlimsup\left|\frac{h_{k+1}}{h_{k}}\right|<1$, then for any $g(t) \in \bigcap_{n} I_{\beta_{n}}\left(H_{0}\right)$ and any $\beta<\frac{1}{2}$, we have $\lim _{k} \frac{g\left(t+h_{k}\right)-g(t)}{h_{k}^{\beta}}=0$ for almost all $t$.

\section{REFERENCES}

[1] Prokhorov, Yu. V., "Convergence of random processes and limit theorems in the theory of probability." Theory of Prob. \& Appl. 1 (1956) pp. 157-214.

[2] Minlos, R. A., "Generalized random processes and their extension to measures." (in Russian) Trudy Moskov, Mat. Obvv. 8 (1959) pp. 497-518.

[3] Kolmogorov, A. N., "A note on the papers of R. A. Minlos and V. Sazonov." Theory of Prob. \& Appl. 4 (1959) pp. 221-223.

[4] Schwartz, L., "Theorie des distributions." Chap. VI. § 5 Paris, Hermann (1951). 